\title{
Vida e morte na atenção primária à saúde: reflexões sobre a vivência do médico de família e comunidade ante a finitude da vida
}

\author{
Life and death in primary care: family and community physician's reflections before the finitude of life
} Vida y muerte en el nivel primario de atención: reflexiones sobre la experiencia del médico familiar con la finitud de la vida

Renata Regina Vieira, Amália Rocha Robortella, Amanda Brólio de Souza, Gustavo Shikanai Kerr, Janaine Aline Camargo de Oliveira

\section{Resumo}

\begin{abstract}
A Atenção Primária à Saúde (APS) possui capacidade para contribuir para que o cuidado ao final da vida seja sistêmico, humanizado e promotor de autonomia. Contudo, prestar assistência em cuidados paliativos requer da equipe um olhar complexo e multidimensional, sobretudo pela proximidade com reflexões sobre morte, perspectivas existenciais, sociais, religiosas e pessoais. Desse modo, perguntas sobre como lidar com as dimensões subjetivas de pacientes, familiares e profissionais surgem como desafio na prática clínica. Sob a perspectiva dos médicos assistentes, relatamos caso de paciente em cuidados paliativos acompanhado por uma equipe de estratégia saúde da família. Ao refletir sobre a dimensão pessoal e existencial dos profissionais, discutimos fatores que favorecem ou dificultam a assistência integral em cuidados paliativos na APS. Observamos que, apesar do desafio da assistência em termos de recursos e tempo, o diálogo na equipe e a abordagem biopsicossocial-espiritual permitiram a ressignificação do processo de cuidado, adoecimento e morte.
\end{abstract}

\begin{abstract}
Primary Care has the ability to enable the end of life care to be systemic, humanized and autonomy promoting. However, working at palliative care requires that the team develop a complex and multidimensional point of view, especially due to the proximity with reflections about death and existential, social, religious and personal perspectives. In this way, questions about how to deal with the subjective dimension of patients, families and professionals emerge as a challenge in clinical practice. Reporting the family physicians' perspectives, we present the case of a patient in palliative care accompanied by a Family Health Strategy team, an unusual scenario for palliative care in Brazil. Thinking about the personal and existential dimensions of the professionals, we discuss factors that favor or hinder the comprehensive care towards the end of a life. We noticed that, despite the challenge of care in terms of resource and time, the dialogue among the team and the biopsychosocial-spiritual approach enabled the redefinition of the process of care, disease and death.
\end{abstract}

\section{Palavras-chave:} Atenção primária à saúde Medicina de família e comunidade Cuidados paliativos

Keywords:

Primary health care Community Family Practice Palliative Care
Como citar: Vieira RR, Robortella AR, Souza AB, Kerr GS, Oliveira JAC. Vida e morte na atenção primária à saúde: reflexões sobre a vivência do médico de família e comunidade ante a finitude da vida. Rev Bras Med Fam Comunidade. 2016;11(38):1-7. http://dx.doi.org/10.5712/rbmfc11(38)1281
Fonte de financiamento: declaram não haver. Parecer CEP: não se aplica. Conflito de interesses: declaram não haver. Procedência e revisão por pares: revisado por pares. Recebido em: 24/02/2016. Aprovado em: 27/09/2016. 


\section{Resumen}

La atención primaria de salud (APS) tiene la capacidad de contribuir con el cuidado al final de la vida de modo sistémico, humanizado y promotor de la autonomía. Sin embargo, un equipo de cuidados paliativos requiere una mirada compleja y multidimensional de sus profesionales, especialmente para hablar sobre la muerte y las perspectivas personales, sociales y religiosas de la vida. Por lo tanto, las preguntas sobre cómo discutir las dimensiones subjetivas de los pacientes, familias y profesionales emergen como un reto en la práctica clínica. Desde la perspectiva de los médicos familiares asistentes, relatamos el caso de un paciente en cuidados paliativos acompañado por un equipo de estrategia de salud de la familia, un escenario poco usual para los cuidados paliativos en Brasil. Desde la reflexión sobre las dimensiones personal y existencial de los profesionales, se discuten los factores que favorecen o dificultan la atención integral de los cuidados paliativos en la APS. Observamos que, a pesar del reto de la asistencia en términos de recursos y tiempo, el diálogo con el equipo y el enfoque biopsicosocial-espiritual permitieron la re-significación del proceso de atención, experiencia de la enfermedad y muerte.
Palabras clave:

Atención Primaria de Salud

Medicina Familiar

y Comunitaria

Cuidados Paliativos

\section{Introdução}

O papel da Atenção Primária à Saúde (APS) na rede de atenção passa pelo primeiro contato, coordenação do cuidado, longitudinalidade e atenção integral. ${ }^{1}$ Prestar assistência contínua, resolutiva e de qualidade exige da APS uma crescente complexidade no cuidado.

Com o envelhecimento e maior prevalência de doenças crônico-degenerativas, cada vez mais, o médico de família de comunidade (MFC) se depara com situações clínicas que seguirão seu curso letal inexoravelmente, provocando diferentes graus de sofrimento físico, emocional, social e espiritual às pessoas e suas famílias.

Perante essas situações, é preciso que a equipe mantenha seu compromisso, prestando assistência de modo a promover qualidade de vida, dignidade e autonomia a essas pessoas. ${ }^{2}$ Esse processo de cuidado integral é chamado de cuidado paliativo "uma abordagem que promove a qualidade de vida, por meio da prevenção e do alívio do sofrimento de natureza física, psicossocial e espiritual". ${ }^{3}$

Cada vez mais cresce o papel da APS na assistência a pessoas em cuidados paliativos. Com isso, as equipes se deparam com a necessidade de desenvolver competências para lidar com a finitude da vida, o sofrimento pela aproximação da morte e o estreitamento dos vínculos com pacientes e familiares. ${ }^{4}$

Este artigo relata de modo narrativo e reflexivo a experiência dos médicos assistentes ao acompanharem paciente em cuidados paliativos devido à neoplasia gástrica com carcinomatose. $\mathrm{O}$ caso foi acompanhado de março de 2014 a fevereiro de 2015, por uma equipe de Estratégia Saúde da Família da rede municipal de São Paulo, na Unidade Básica de Saúde Parque Regina, campo de estágio do Programa de Residência em Medicina de Família e Comunidade do Hospital Israelita Albert Einstein. A publicação teve consentimento livre e esclarecido da família e foi isentada de parecer pelo Conselho de Ética da Instituição em 9 de novembro de 2015.

\section{Narrativa do caso}

Conhecê-la foi como ver uma andorinha insegura no ninho... Vinha emagrecida, pele marcada pelo esmorecer e com olhos que ansiavam por entender: "O que se passava com aquela dor no estômago?". Havia eliminado sangue... Não foi preciso ser sagaz para compreender os sinais do corpo físico. A endoscopia digestiva foi certeira e a biópsia não deixou dúvidas: adenocarcinoma gástrico penetrando paredes e antro do órgão que alimenta a vida. 
Recebeu o diagnóstico sozinha, com um pouco de entendimento e um muito de fé. Com uma segurança que não tínhamos, falávamos-lhe sobre os próximos passos. A família se aproximou após o diagnóstico. Vinham do interior de Pernambuco para buscar sobreviver em São Paulo. Viviam em um pequeno cortiço... casa pequena, simples, mas sempre muito bem cuidada. Sofriam por saber que não dispunham de recursos para auxiliar no tratamento. Juntos, aguardaram ansiosos a cirurgia nunca realizada e a quimioterapia da qual esperavam a cura. Não faltaram preces, visitas do pastor evangélico e, claro, a garrafada da vizinha: conhaque, mel e babosa já curaram muitas feridas no estômago.

Seis meses se foram céleres. Nossa equipe, sem receber informações do centro de tratamento, não sabia bem o que esperar. A família agora estava insegura. A andorinha já não tinha mais forças para voar. Emagrecia, passava a ter dores permanentes no epigastro, já não tolerava o alimento. O que mais era possível fazermos? Fomos à primeira visita em seu ninho.

Já estava com sonda nasoentérica, mas não aceitava a dieta. O marido brigava para forçá-la a comer: queria seu bem e não era por mal que negava a doença. Além disso, a pouca instrução dos anos primários lhe traziam dificuldade de lidar com a infusão do preparado industrial. Mas não... A questão da hiporexia era a falta de fome de sua alma. Foi a primeira vez em que falamos sobre o significado de tantos acontecimentos: "Não quero mais comer, pois tudo que eu como, serve de alimento primeiro para essa ferida, que só cresce...". Primeiro mostrou-nos sua ideia sobre o que "alimentava a doença" e, depois, a nova tomografia.

Pudemos perceber o estadiamento tumoral e compreendemos o adiamento da cirurgia. Nenhum médico havia sido claro sobre seu prognóstico. Mas a vida é verdade que não se esconde: ela começava a sentir sua despedida. Sim... Cabia à APS coordenar o cuidado: acompanhar a pessoa em seu diagnóstico e tratamento, curativo ou não. Vimo-nos diante da incerteza sobre nosso papel: sim, ela precisava de cuidados paliativos. Se nós não estivéssemos ali para fazê-lo, ninguém o faria.

Na sala pequenina, uma troca de olhar entre profissionais disse tudo: médicos de família e comunidade se identificam pelo seu compromisso com a pessoa. Cumpriríamos nosso papel: seríamos os contra-regras que fariam o possível para que a andorinha estrelasse seu último verão de modo autônomo e pleno.

Marido, filha e cunhado sabiam que tinhamos algo sério a dizer enquanto o netinho brincava no chão. Conversamos com eles sobre as crenças: qual seria o propósito da doença? Qual o propósito da vida? Ela se mostrava serena, enquanto o marido, com o último balbucio da barganha, perguntava: "Doutora, você já viu pessoas como ela se curarem, não viu?". A dignidade deles merecia a nossa honestidade.

Em meio à dor, já não apenas física, vimos a força do diálogo a ressignificar aquela narrativa: juntos revisitamos a história da doença, antes vista como punição de Deus a uma mulher que não pecara, e agora sendo reconstruída como uma forma de ela provar sua fé para a entrada no "Reino dos Céus".

Com a aceitação, ela se fortaleceu. Em sua força e exemplo, ela pareceu chamar ao "testemunho" aqueles que conviviam com ela. A filha, antes chorosa, vinha agora disposta a auxiliar a mãe. O marido aceitava melhor suas escolhas. Nossa equipe também se mobilizou: residentes, preceptora, enfermeira, auxiliares e agente comunitária se revezavam em visitas duas vezes por semana, orientando os cuidados, mas também promovendo momentos de socialização e resgate de lembranças. Da morfina à oração com a família na sala, pudemos compartilhar com eles momentos ímpares, compreendendo que nossa humanidade era única, a despeito de valores pessoais. 
Com poucos recursos, os dois meses que se seguiram não foram fáceis. Mas foram significativos! A dieta, adaptada pela nutricionista, era mínima pelo estado consumptivo, mas já melhor tolerada. A morfina permitia conforto necessário para os últimos atos: reatar com o filho distante há dois anos e compreender que guardar rancores já não fazia mais sentido. As orações com o grupo da igreja que a ACS e a filha mobilizaram traziam plenitude para a alma. Celebrou o último natal com visitas longínquas de entes queridos e o sabor das poucas guloseimas que conseguia ingerir. E, nos derradeiros instantes da peça de sua vida, um pouco de morfina, uma cadeira de rodas, a mobilização dos amigos da igreja e uma grande dose de amor permitiram que ela fosse "batizada nas águas", aos moldes de sua crença religiosa.

Em nossa última visita a seu ninho, a andorinha trazia olhos cintilantes. Sim, brilhavam como sua paz ao se despedir: "Agora já estou pronta para descansar". A família sofria, mas tirava da crise as estratégias para seguir em frente. Não faleceu em sua casa.

Nossa falta de recursos humanos e segurança para passar para à família, unidas a pouca instrução deles, não nos permitiram. No entanto, manteve-se soberana: seu desejo de não receber procedimentos invasivos, pactuado previamente, foi compreendido e respeitado nas 48 horas que passou no leito hospitalar. Ela não partiu. Ela voou para o inominável da Vida levando um pouco de nós e deixando muito de si. Seu exemplo nos trouxe a vontade de servir cada vez mais ao Cuidado para que outras andorinhas possam, também, voar!

\section{Discussão}

Prestar assistência paliativa requer olhar complexo e multidimensional sobre a pessoa e seu sistema familiar. Nessa etapa de vida, é preciso cuidar da dor total, vivenciada de modo único por cada pessoa, em suas dimensões física, emocional, sociofamiliar e espiritual. ${ }^{5}$ Nesse sentido, cabem reflexões sobre sofrimento e morte, sob perspectivas existenciais, sociais, religiosas e pessoais. ${ }^{6}$ Essa construção dialógica considera a experiência de vida da pessoa e familiares, traz à tona valores e crenças, e exige o olhar sobre a dimensão espiritual do ser humano. ${ }^{7}$

Dada sua importância nos cuidados paliativos, cabe trazermos o conceito de espiritualidade. A espiritualidade é a dimensão em que obtemos significado, conexão, conforto e paz para nossas vidas. Pode ser buscada na religião, como ocorre mais comumente, mas também na música, arte, natureza ou em valores e princípios pessoais ou científicos. ${ }^{8}$ Essa busca passa pelo campo pessoal de reflexão, relacionamentos e pela dimensão do sagrado ou transcendente. ${ }^{9}$

Tratar sobre essas questões, muitas vezes distantes da formação médica, pode ser um desafio. Um estudo norueguês com MFC observou que diferentes fatores influenciam o processo de lidar com a morte de um paciente: os relacionados com o médico, ambiente e o paciente. ${ }^{4}$ Relacionaram-se ao paciente a proximidade e intensidade da relação terapêutica, bem como o tipo de morte (abrupta, humanizada ou invasiva).

Quanto ao ambiente, destacaram-se o contexto cultural e religioso, experiências prévias das pessoas, manejo do luto e educação sobre a morte. Já dentre os médicos, observou-se a vivência de profundas emoções ao prestarem esse tipo de cuidado, tais como culpa, medo, raiva, impotência, tristeza, distanciamento emocional e alívio. Esse contato também traz à tona vivências pessoais, como experiências de perda ou adoecimento, e questionamentos pessoais sobre morte e o significado ou propósito da vida. 
Cuidados paliativos visam promover qualidade de vida. Na experiência com o caso, houve controle de sintomas (dor, plenitude gástrica e hiporexia), abordagem familiar, mobilização de rede social (família, igreja evangélica, grupo de oração), apoio espiritual e abordagem antecipada do luto. Essas iniciativas de cuidado integral impactaram na qualidade de vida da paciente, tanto prevenindo o adoecimento da família quanto pela marcante melhora de performance funcional.

A funcionalidade de pacientes com neoplasias avançadas pode ser avaliada a partir escala de performance de Karnofsky (EPK), método descrito na década de 50 que classifica o status funcional em 11 categorias, variando de 100\% (asintomático, sem evidência de doença) a 0 (morte), conforme o grau de dependência para atividades. ${ }^{10}$

Com as ações em cuidados paliativos implementadas, a paciente, inicialmente em Karnofsky 40 (mais de $50 \%$ do tempo acamada, requerindo atenção constante), teve melhora funcional para Karnofsky 60 (apta ao autocuidado, requerindo assistencia ocasional, por exemplo, com manuseio da dieta enteral). Nas duas últimas semanas de vida, houve novo declínio funcional até 40 , porém com paciente mantendo-se orientada, em fase de aceitação e capaz de conversar sobre sua percepção subjetiva de plenitude espiritual. Nos últimos dois dias de vida, com o início de dispneia, a paciente evoluiu para 20 pontos (agravamento clínico com necessidade de cuidados contínuos) e foi hospitalizada até o óbito.

A promoção de um cuidado digno e transformador para a família trouxe grande satisfação à equipe de ESF. Sentimentos de angústia e impotência percebidos inicialmente pela equipe, pela dificuldade com recursos e falta de informações sobre prognóstico, puderam ser amenizados a partir da abordagem biopsicossocial-espiritual. Percebemos que tecnologias leves, como comunicação centrada na pessoa, abordagem familiar e espiritual, diretivas antecipadas e manejo do luto foram diferenciais para o bem-estar da família e motivaram a equipe quanto à qualidade e impacto do cuidado prestado.

Aprender a ressignificar a visão profissional, indo do sofrimento para a valorização da vida, foi um passo essencial, construído a partir da mobilização de recursos pessoais de enfrentamento e resiliência. Assim, a dimensão narrativa de significado ganha foco no âmbito do cuidado da equipe, o que torna cada experiência de morte única e parte de um sistema de aprendizado contínuo. ${ }^{11}$

Ainteração médico-paciente é influenciada por valores da pessoa e do profissional. Para garantir esse vínculo, o médico deve trabalhar seu autoconhecimento, tornando-se mais consciente dos processos de transferência e contratransferência. ${ }^{12} \mathrm{~A}$ dificuldade dos profissionais de saúde lidarem com sua dimensão pessoal é natural, contudo, corre-se o risco de que as questões sobre sofrimento e terminalidade sejam negligenciadas. ${ }^{6}$

Outro aspecto preocupante é o risco de sobrecarga profissional. Dearmond ${ }^{13}$ descreve que já são bem documentados burnout e fadiga de compaixão em profissionais paliativistas. Por outro lado, há associação entre autocuidado e resiliência dos profissionais com menores níveis de burnout e maiores níveis de compaixão e satisfação. Em seu estudo, observou que profissionais que trabalham com a filosofia de cuidados paliativos poderiam ter transformações pessoais, maior senso de consciência e autotranscendência.

No contexto de porta de entrada e longitudinalidade da APS, a demanda por cuidados paliativos está presente no cotidiano do MFC, mas pode ser limitada pela insegurança dos profissionais. Realizar essa abordagem possibilitou criar vínculo e confiança com paciente, familiares e equipe multiprofissional, 
permitindo o diálogo claro sobre a finitude. Essa postura reduziu a sobrecarga da equipe e permitiu a vivência dos diferentes estágios do luto. A equipe da ESF pôde fechar esse ciclo com a certeza de ter oferecido um processo de morte digno.

\section{Conclusão}

Abordar situações de perda e morte na APS é um grande desafio em termos de recursos e disponibilidade. Contudo, a mobilização da equipe e a abordagem biopsicossocial-espiritual se mostraram potencialidades interessantes para a promoção da qualidade de vida nesse contexto.

\section{Referências}

1. Starfield B. Atenção primaria: equilíbrio entre necessidades de saúde, serviços e tecnologia. Brasília:UNESCO, Ministério da Saúde; 2004. 726 p.

2. Silva MLSR. O papel do profissional da Atenção Primária à Saúde em cuidados paliativos. Rev Bras Med Fam Comunidade. 2014;9(30):45-53. DOI: http://dx.doi.org/10.5712/rbmfc9(30)718

3. World Health Organization. Palliative care for older people: better practices. Copenhagen: WHO; 2011.

4. Verhoeven AA, Schuling J, Maeckelberghe EL. The death of a patient: a model for reflection in GP training. BMC Fam Pract. 2011;12:8. DOI: http://dx.doi.org/10.1186/1471-2296-12-8

5. Clark D. "Total pain", disciplinary power and the body in the work of Cicely Saunders, 1958-1967. Soc Sci Med. 1999;49(6):727-36. DOI: http://dx. doi.org/10.1016/S0277-9536(99)00098-2

6. Cassell EJ. Diagnosing suffering: a perspective. Ann Intern Med. 1999;131(7):531-4. DOI: http://dx.doi.org/10.7326/00034819-131-7-199910050-00009

7. Seccareccia D, Brown JB. Impact of spirituality on palliative care physicians: personally and professionally. J Palliat Med. 2009;12(9):805-9. DOI: http://dx.doi.org/10.1089/jpm.2009.0038

8. Anandarajah G, Hight E. Spirituality and medical practice: using the HOPE questions as a practical tool for spiritual assessment. Am Fam Physician. 2001;63(1):81-9.

9. Puchalski CM. The FICA Spiritual History Tool \#274. J Palliat Med. 2014;17(1):105-6. DOI: http://dx.doi.org/10.1089/jpm.2013.9458

10. de Borja MT, Chow E, Bovett G, Davis L, Gillies C. The correlation among patients and health care professionals in assessing functional status using the Karnofsky and Eastern Cooperative Oncology Group Performance Status scales. Support Cancer Ther. 2004;2(1):59-63. DOI: http://dx.doi.org/10.3816/SCT.2004.n.024

11. Jones AH. Narrative based medicine: narrative in medical ethics. BMJ. 1999;318(7178):253-6. DOI: http://dx.doi.org/10.1136/bmj.318.7178.253

12. Balint M. O médico, seu paciente e a doença. $1^{\text {a }}$ ed. Rio de Janeiro: Atheneu; 1988.

13. Dearmond IM. The psychological experience of hospice workers during encounters with death. Omega (Westport). 2012-2013;66(4):281-99. DOI: http://dx.doi.org/10.2190/OM.66.4.a 
Renata Regina Vieira. Hospital Israelita Albert Einstein.

São Paulo, SP, Brasil. renata_reginav@yahoo.com.br

Amália Rocha Robortella. Hospital Israelita Albert Einstein. São Paulo, SP, Brasil. amaliarob @ hotmail.com

Amanda Brólio de Souza. Hospital Israelita Albert Einstein. São Paulo, SP, Brasil. amandabrolio@ hotmail.com

Gustavo Shikanai Kerr. Hospital Israelita Albert Einstein. São Paulo, SP, Brasil. gustavo.kerr@einstein.br

Janaine Aline Camargo de Oliveira. Grupo de Trabalho em Saúde e Espiritualidade da SBMFC; Hospital Israelita Albert Einstein. São Paulo, SP, Brasil. jancamargo@ hotmail.com (Autora correspondente) 